Marquette University

e-Publications@Marquette

Mechanical Engineering Faculty Research and

Publications

Mechanical Engineering, Department of

$4-2008$

\title{
Admittance Selection Conditions for Frictionless Force-Guided Assembly of Polyhedral Parts in Two Single-Point Principal Contacts
}

Shuguang Huang

Marquette University, shuguang.huang@marquette.edu

Joseph M. Schimmels

Marquette University, joseph.schimmels@marquette.edu

Follow this and additional works at: https://epublications.marquette.edu/mechengin_fac

Part of the Mechanical Engineering Commons

\section{Recommended Citation}

Huang, Shuguang and Schimmels, Joseph M., "Admittance Selection Conditions for Frictionless ForceGuided Assembly of Polyhedral Parts in Two Single-Point Principal Contacts" (2008). Mechanical

Engineering Faculty Research and Publications. 81.

https://epublications.marquette.edu/mechengin_fac/81 
Marquette University

\title{
e-Publications@Marquette
}

\section{Mechanical Engineering Faculty Research and Publications/College of Engineering}

This paper is NOT THE PUBLISHED VERSION; but the author's final, peer-reviewed manuscript. The published version may be accessed by following the link in the citation below.

IEEE Transactions on Robotics, Vol. 24, No. 2 (April 2008): 461-468. DOI. This article is (C) Institute of Electrical and Electronics Engineers (IEEE) and permission has been granted for this version to appear in e-Publications@Marquette. Institute of Electrical and Electronics Engineers (IEEE) does not grant permission for this article to be further copied/distributed or hosted elsewhere without the express permission from Institute of Electrical and Electronics Engineers (IEEE).

\section{Admittance Selection Conditions for Frictionless Force-Guided Assembly of Polyhedral Parts in Two Single-Point Principal Contacts}

\author{
Shuguang Huang \\ Marquette Univ., Milwaukee \\ Joseph M. Schimmels \\ Marquette Univ., Milwaukee
}

\begin{abstract}
:
The admittance of a manipulator can be used to improve robotic assembly. If properly selected, the admittance will regulate a contact force and use it to guide the parts to proper positioning. In previous work, procedures for selecting the appropriate admittance for single principal contact (PC) cases were identified. This paper extends this research for some of the two PC cases-those for which each contact occurs at a single point. The conditions obtained ensure that the motion that results from frictionless contact always instantaneously reduces part misalignment. We show that, for bounded misalignments, if an admittance satisfies the misalignment-reducing conditions at a
\end{abstract}


finite number of contact configurations, then the admittance will also satisfy the conditions at all intermediate configurations.

Keywords: spatial admittance, Assembly, compliance selection, multiple-point contact

\section{SECTION I. Introduction}

In robotic assembly, admittance control has been used to provide force regulation and force guidance. The admittance changes contact forces into changes in the velocity of the body held by the manipulator. If properly designed, the manipulator admittance will cause the held part to move toward the desired position, thus correcting misalignment. Here, procedures for selecting the appropriate manipulator admittance for polyhedral part assembly subtasks are identified.

A simple form of admittance, a linear admittance control law [1] is considered. For spatial applications, this admittance behavior has the form

$$
\mathbf{v}=\mathbf{v}_{0}+\mathbf{A w}(1)
$$

where $\mathbf{v}_{0}$ is the nominal twist (a six-vector), $\mathbf{w}$ is the contact wrench (force and torque) measured in the body frame (a six-vector), $\mathbf{A}$ is the admittance matrix (a $6 \times 6$ matrix), and $\mathbf{v}$ is the motion of the body.

When the held body is in contact with its mating part, the contact force will yield a motion by control law (1). An admittance design objective is that it achieves force assembly, i.e., for any given contact state, the admittance always leads to a motion that reduces the misalignment. Since the error-reducing motion is generated by the contact force, no sensors or actuators are needed. A desired admittance can be realized with either a passive compliant mechanism mounted on the end-effector of the manipulator [2] or through robot control [3].

Similar to previous work [4]-[5][6], in this paper, we consider a measure of error based on the Euclidean distance between an arbitrarily chosen single (fixed) point on the held body and its location when properly positioned. Since there is no "natural metric" for finite spatial error of a body, the error measure used is body-specific [7]. Using this measure of misalignment, the error-reduction condition can be expressed mathematically as

$$
\mathbf{d}^{T} \mathbf{v}=\mathbf{d}^{T}\left(\mathbf{v}_{0}+\mathbf{A w}\right)<0(2)
$$

where $\mathbf{d}$ (a six-vector for spatial motion) is the line vector from the selected point at its properly mated position to its current position and $\mathbf{w}$ is the contact wrench. Force assembly [1] requires that, at each possible misalignment, the contact force yields a motion that reduces the misalignment. As such, to achieve an error-reducing motion for a specific contact state, condition (2) must be satisfied for all possible misalignments in that contact state. 
Since there are an infinite number of configurations within a given contact state, it is impossible to impose the error-reduction condition (2) on all configurations. Our objective is to identify a set of conditions that are imposed on the admittance at a finite number of configurations to ensure error-reducing motion for all configurations associated with a given contact state. Once these conditions are established, they can be used to guide the search for an appropriate admittance.

Others addressed the selection of an admittance for assembly [8]-[9][10][11]. The forceassembly approach used in this paper differs from the others' in that: 1) it applies to any two polyhedral parts and 2) ensures that error-reducing motion is achieved.

This paper is an extension of our previous work [6] in which sufficient conditions imposed on an admittance were used to ensure the force assembly of polyhedral parts for each of the six single principal contact (PC) cases. In this paper, conditions on an admittance for parts with two single-point PCs are identified. The overall admittance selection strategy is based on problem decomposition. Since for polyhedral parts, the set of assembly contacts can be decomposed into a set of contact states, the admittance for the assembly can be selected by selecting the appropriate admittance for each contact state. If sufficient conditions for each contact state in the assembly task are satisfied simultaneously, successful assembly can be ensured without having to determine in real time which contact state is being encountered.

In the two-PC cases, due to geometric constraints, the generalized coordinates used in [6] for each contact point are now coupled. This coupling is highly nonlinear and part geometryspecific. To address this in a rigorous and geometry-independent way, we consider the coupling that occurs between the two contact forces, but ignore the coupling of the two sets of coordinates. In doing this, a space of contact configurations larger than possible is considered. Due to the coupling of the two contact forces, the two sets of conditions developed independently for each of the two single-PC cases are not equivalent to the set of conditions developed for the corresponding two-PC case. Thus, in developing the admittance selection conditions for the two-PC cases, the contact forces associated with each contact are considered simultaneously.

For two point contact, if we denote $\mathbf{w}_{i}$ as the unit wrench associated with contact point $i$ and denote $\phi_{i}$ as the corresponding magnitude, and

$$
\mathbf{W}=\left[\mathbf{w}_{1}, \mathbf{w}_{2}\right] \in \mathbb{R}^{6 \times 2}, \phi=\left[\phi_{1}, \phi_{2}\right]^{T} \in \mathbb{R}^{2}
$$

then, the total contact wrench is

$$
\mathbf{w}=\mathbf{W} \phi .
$$

Using the reciprocal condition [12], it can be shown that

$$
\phi=-\left[\mathbf{W}^{T} \mathbf{A} \mathbf{W}\right]^{-1} \mathbf{W}^{T} \mathbf{v}_{0} \cdot(3)
$$

The error reduction function can be written as 


$$
F_{\text {er }}=\mathbf{d}^{T}\left(\mathbf{v}_{0}+\mathbf{A W} \phi\right) \text {. (4) }
$$

As shown in [4], the error reduction function can be equivalently written as

$$
F_{\text {er }}=\left(\mathbf{d}^{T} \mathbf{v}_{0}\right) \operatorname{det}\left(\mathbf{W}^{T} \mathbf{A W}\right)-\mathbf{d}^{T} \mathbf{A} \mathbf{W}\left[\mathbf{W}^{T} \mathbf{A} \mathbf{W}\right]^{*} \mathbf{W}^{T} \mathbf{v}_{0}(5)
$$

where $\left[\mathbf{W}^{T} \mathbf{A W}\right]^{*}$ denotes the adjugate matrix of $\mathbf{W}^{T} \mathbf{A W}$.

Since $\mathbf{d}$ and $\mathbf{w}$ are position-dependent, $F_{\text {er }}$ is a function of configuration. For bounded configuration variations, those at the extremes are of interest. In this paper, a set of conditions on the admittance is derived for each contact state such that, if satisfied at the "extremal" configurations, these conditions ensure error-reducing motion for all configurations associated with a given contact state.

\section{SECTION II. Configuration Description}

The contact states studied here involve two of the single-point contact principal contacts (or PCs) [13] obtained for polyhedral parts. Single-point contact PCs are of three types: "face vertex" $(\{f-v\}))$ contact, "vertex face" $(\{v-f\})$ contact, and "edge-edge cross" $\left(\{e-e\}_{c}\right)$ contact. Of the six combinations of two single-point contact PCs, four cases are addressed in detail. The extension of these procedures to the remaining two cases is only described.

\section{A. Single-PC States}

For single-point contact states, the body can translate in the plane of contact and rotate about the contact point in any direction. As such, five variables describe the relative configuration of the bodies (the relative position of the contact point using two translational variables and the relative orientation using three rotational variables).

The description of configuration variation for single-point PCs was established in [4] and is briefly reviewed next.

\section{Orientational Variation}

A unit vector $\mathbf{u}$, indicating the direction of the rotational axis, and a scalar $\theta$, indicating the rotation angle are used to describe orientation variation. The relative orientation of the rigid body can be described by a $3 \times 3$ orthogonal matrix $\mathbf{R}$ by Rodrigues' formula [14]

$$
\mathbf{R}(\mathbf{u}, \theta)=\cos \theta \mathbf{I}+(1-\cos \theta) \mathbf{u u}^{T}+\sin \theta[\mathbf{u} \times](6)
$$

where $[\mathbf{u} \times]$ denotes the antisymmetric matrix associated with the cross product operation involving $\mathbf{u}$. 
For a finite variation, we place bounds on the maximum angular magnitude $0 \leq \theta \leq \theta_{M}$ but place no bounds on the direction of the rotation axis $\mathbf{u}$.

\section{Translational Variation}

For bodies in contact at a single point, the location of the contact point can be described by two parameters $\delta=\left(\delta_{1}, \delta_{2}\right)$. The meaning of these variables for each of the principle contacts considered is described next.

For face-vertex $(\{f-v\})$ contact, a two-dimensional coordinate frame $O_{b}$ is established on the held body in the plane of the contact face. Two orthogonal coordinates $\left(\delta_{1}, \delta_{2}\right)$ are used to describe translational variation of the rigid body within this contact state as shown in Fig. 1(a).

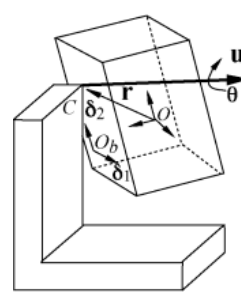

(a)

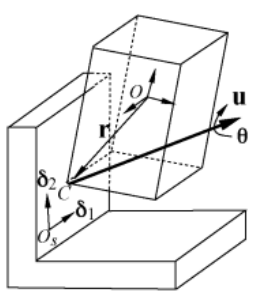

(b)

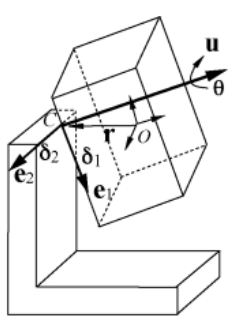

(c)

Fig. 1. Configuration variables for single-point PCs. (a)Face-vertex contact. (b) Vertex-face contact. (c) Edge-edge cross contact.

For vertex-face $(\{v-f\})$ contact, a 2-D coordinate frame $O_{s}$ is established on the stationary part in the plane of the contact face. Again, two orthogonal coordinates $\left(\delta_{1}, \delta_{2}\right)$ are used to describe the translational variation of the rigid body within this contact state as shown in Fig. 1(b).

For edge-edge cross $\left(\{e-e\}_{c}\right)$ contact, two translational non-orthogonal coordinates $\left(\delta_{1}, \delta_{2}\right)$ are chosen to describe translational variation along edges $\mathbf{e}_{1}$ and $\mathbf{e}_{2}$ as shown in Fig. 1(c).

Since finite configuration variation is considered for each contact state, the variation of each $\delta_{i}$ is bounded. By appropriately choosing the coordinate origin (at a central location of contact), the bounds for $\delta_{i}$ can be written as

$$
-\Delta_{i} \leq \delta_{i} \leq \Delta_{i}, i=1,2
$$

In summary, configuration variation for each single-point contact state is given by $\mathbf{q}=$ $\left(\delta_{1}, \delta_{2}, \mathbf{u}, \theta\right)$. 


\section{B. Two-PC States}

When the two-PC case is considered, the DOF of the body is reduced. The configuration of the body can be determined by one of the two sets of the generalized coordinates associated with the given two single-point contact PCs. In the two sets of coordinates $\mathbf{q}_{1}$ and $\mathbf{q}_{2}$, the orientational variables $(\mathbf{u}, \theta)$ are the same, while the translational variables are not the same and are not independent. The relationship between the translational variables $\delta_{1}=\left(\delta_{11}, \delta_{12}\right)$ and $\delta_{2}=\left(\delta_{21}, \delta_{22}\right)$ are determined by the geometric constraints associated with contact. In the 4-D subspace of $\left(\delta_{1}, \delta_{2}\right)$, the constrained configuration space is a lower dimensional manifold. Thus, it would be sufficient if the error-reduction conditions were satisfied only on the manifold. Since the constraint equations are nonlinear and complicated, the construction of this manifold or reduction in its dimensionality (i.e., finding a set of independent variables) is geometry-specific and very difficult.

In order to obtain sufficient conditions (without calculating and solving the equations associated with the constraints), it is required that the error-reduction conditions are satisfied not only on the manifold but also in the entire range of variation considered. Thus, all combinations of $\delta_{i j}$ in the range will be considered regardless how they are related. Therefore, only the ranges of the four translational variables are considered and each $\delta_{i j}$ is treated as independent within each variable's range. In doing this, the error-reduction conditions are enforced in a larger than geometrically possible configuration space. As such, the obtained conditions are conservatively sufficient for all configurations in the range considered.

As stated previously, it is important to note that, although configuration variables $\delta_{i j}$ are treated as independent, the contact forces at each contact are dependent. This means that, in some configurations, contact between part features is assumed to exist despite the fact that either contact would not occur or parts would interpenetrate for this configuration of the specific parts. For all two-PC cases considered, the coupling of the contact forces is explicitly addressed in the calculation of their magnitudes (3). It should also be noted that the errorreduction function, in general, is a nonlinear function of generalized coordinates.

\section{SECTION III. Sufficient Conditions for $\{f-v, f-v\}$ Contact}

As shown in Section II, the relative configuration of the bodies in face-vertex contact is described by the two sets of translational variables $\left(\delta_{11}, \delta_{12}\right),\left(\delta_{21}, \delta_{22}\right)$ and the orientational variables $(\mathbf{u}, \theta)$. We prove that, if an admittance matrix $\mathbf{A}$ satisfies a set of conditions at the "boundary" points of the configurations, then the $\mathbf{A}$ matrix ensures error-reducing motion for all intermediate configurations $\delta_{i j} \in\left[-\Delta_{i j}, \Delta_{i j}\right]$ and $\theta \in\left[0, \theta_{M}\right]$ (regardless of the direction of rotation).

To obtain these results, the error-measure vector $\mathbf{d}$ and contact forces are expressed as functions of the configuration variables. Next, the bounds of the contact force magnitudes are determined by evaluating the functions at the boundary configurations. Then, using the bounds 
of the force magnitudes, a set of conditions on the admittance for error-reducing motion is obtained.

\section{A. Error-Measure Vector and Contact Forces}

For a single $\{f-v\}$ PC $i$, the contact wrench and the error-measure vector $\mathbf{d}$ can be expressed as functions of $\left(\delta_{i 1}, \delta_{i 2}, \mathbf{u}, \theta\right)$. As shown in [4], when the held body rotates relative to the fixtured body about the contact point, the description of the contact wrench does not change in a body-based coordinate frame. When the held body translates relative to the fixtured body, the description of the contact wrench changes in a body-based coordinate frame because the contact point changes (although its direction is constant). Thus, the contact wrench is a function involving only the translational variables $\left(\delta_{i 1}, \delta_{i 2}\right)$.

As shown in Fig. 2(a), for an arbitrary face-vertex contact, the direction of the surface normal is constant in the body frame while the position vector of the contact point $\mathbf{r}$ varies. For arbitrary $\left(\delta_{i 1}, \delta_{i 2}\right)$, using the same approach presented in [4], $\mathbf{r}_{i}$ can be expressed as

$$
\mathbf{r}_{i}=\mathbf{r}_{0 i}+\delta_{i 1} \mathbf{b}_{i 1}+\delta_{i 2} \mathbf{b}_{i 2}(7)
$$

where $\mathbf{r}_{0 i}$ is the position vector from the body frame's origin $O$ to the origin of the centrally located coordinate frame $O_{b i}$, and $\mathbf{b}_{i 1}$ and $\mathbf{b}_{i 2}$ are unit vectors along the two axes of coordinate frame $O_{b i}$ (constant in body frame). The unit wrench corresponding to the surface normal is

$$
\mathbf{w}_{i}=\left[\begin{array}{c}
\mathbf{n}_{i} \\
\mathbf{r}_{i} \times \mathbf{n}_{i}
\end{array}\right]
$$

Note that in the body frame, the direction of $\mathbf{w}_{i}$ is constant while the last component (the moment term) is a linear function of $\delta_{i j}$.

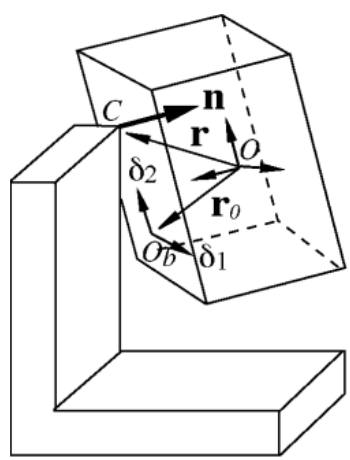

(a)

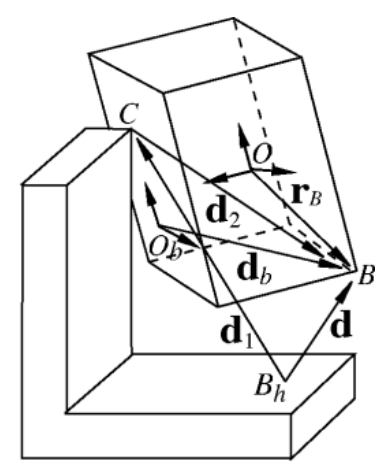

(b)

Fig. 2. Face-vertex contact and the coordinate frame.

The line vector $\mathbf{d}$ associated with error reduction is also a function of configuration. Since the configuration can be represented by $\left(\delta_{11}, \delta_{12}, \mathbf{u}, \theta\right)$ or $\left(\delta_{21}, \delta_{22}, \mathbf{u}, \theta\right)$, either one can be chosen to express the vector $\mathbf{d}$. 
Let $B$ be the point selected for the error measure and $B_{h}$ be the position of $B$ when the body is properly mated [see Fig. 2(b)], and let $\mathbf{d}^{\prime}$ be the 3-D position vector for error measure from $B_{h}$ to $B$ (the first three elements of the line vector $\mathbf{d}$ ). Using the set of variables $\left(\delta_{11}, \delta_{12}, \mathbf{u}, \theta\right)$, it is shown [4] that $\mathbf{d}^{\prime}$ can be expressed as

$$
\mathbf{d}^{\prime}(\delta)=\mathbf{d}_{1}^{\prime}+\mathbf{d}_{b}^{\prime}-\delta_{1}^{\prime}
$$

where $\mathbf{d}_{1}^{\prime}$ is the position vector from $B_{h}$ to the contact point $C, \mathbf{d}_{b}^{\prime}$ is the position vector from the frame origin $O_{b}$ to point $B$ (constant in body frame), and

$$
\delta_{1}^{\prime}=\delta_{11} \mathbf{b}_{11}+\delta_{12} \mathbf{b}_{12} \text {. }
$$

Again note that $\mathbf{d}_{1}^{\prime}$ is constant in the global coordinate frame while $\mathbf{b}_{i}$ and $\mathbf{d}_{b}^{\prime}$ are constant in the body frame. Thus, for an arbitrary orientation $(\mathbf{u}, \theta)$ and $\delta_{1 j} \in\left[-\Delta_{1 j}, \Delta_{1 j}\right]$, the errormeasure three-vector $\mathbf{d}^{\prime}$ is a function of $(\mathbf{u}, \theta)$ and $\delta_{1 j}$ having the form

$$
\mathbf{d}^{\prime}(\mathbf{u}, \theta, \delta)=\mathbf{R} \mathbf{d}_{1}^{\prime}+\mathbf{d}_{b}^{\prime}-\delta_{1}^{\prime}
$$

where $\mathbf{R}$ is the rotation matrix having the form of $(6)$.

The line vector associated with $\mathbf{d}^{\prime}$ can be calculated as

$$
\mathbf{d}(\delta, \theta)=\left[\begin{array}{c}
\mathbf{R} \mathbf{d}_{1}^{\prime} \\
\mathbf{r}_{B} \times \mathbf{R} \mathbf{d}_{1}^{\prime}
\end{array}\right]+\left[\begin{array}{c}
\mathbf{d}_{b}^{\prime} \\
\mathbf{r}_{B} \times \mathbf{d}_{b}^{\prime}
\end{array}\right]-\left[\begin{array}{c}
\delta_{1}^{\prime} \\
\mathbf{r}_{B} \times \delta_{1}^{\prime}
\end{array}\right] \text { (9) }
$$

where $\mathbf{r}_{B}$ is the position vector from the body frame origin $O$ to the error measure point $B$ (constant in body frame).

If we denote

$$
\mathbf{W}=\left[\mathbf{w}_{1}, \mathbf{w}_{2}\right] \in \mathbb{R}^{6 \times 2}
$$

then, using (3), the force magnitudes can be determined:

$$
\begin{aligned}
& \phi_{1}=-\frac{\left(\mathbf{w}_{2}^{T} \mathbf{A} \mathbf{w}_{2}\right)\left(\mathbf{w}_{1}^{T} \mathbf{v}_{0}\right)-\left(\mathbf{w}_{1}^{T} A \mathbf{w}_{2}\right)\left(\mathbf{w}_{2}^{T} \mathbf{v}_{0}\right)}{\operatorname{det}\left(\mathbf{W}^{T} \mathbf{A W}\right)} \\
& \phi_{2}=-\frac{\left(\mathbf{w}_{1}^{T} \mathbf{A} \mathbf{w}_{1}\right)\left(\mathbf{w}_{2}^{T} \mathbf{v}_{0}\right)-\left(\mathbf{w}_{1}^{T} A \mathbf{w}_{2}\right)\left(\mathbf{w}_{1}^{T} \mathbf{v}_{0}\right)}{\operatorname{det}\left(\mathbf{W}^{T} \mathbf{A W}\right)} .
\end{aligned}
$$

\section{B. Bounds of the Contact Force Magnitude}

In order to obtain the bounds of contact force magnitude, first the denominator of $(10)$ and (11) is considered. Since $\mathbf{A}$ is a symmetric positive semidefinite (PSD) matrix, A can be decomposed as

$$
\mathbf{A}=\mathbf{H}^{T} \mathbf{H} .
$$

If we denote $\mathbf{W}_{h}=\mathbf{H W}=\left[\mathbf{w}_{h 1}, \mathbf{w}_{h 2}\right] \in \mathbb{R}^{2 \times 6}$, then 


$$
\operatorname{det}\left(\mathbf{W}^{T} \mathbf{A} \mathbf{W}\right)=\left(\mathbf{w}_{h 1}^{T} \mathbf{w}_{h 1}\right)\left(\mathbf{w}_{h 2}^{T} \mathbf{w}_{h 2}\right)-\left(\mathbf{w}_{h 1}^{T} \mathbf{w}_{h 2}\right)^{2} .
$$

Note that $\mathbf{w}_{h 1}$ and $\mathbf{w}_{h 2}$ are six-vectors. If we denote $a_{k}$ and $b_{k}$ as the $k^{\text {th }}$ components of $\mathbf{w}_{h 1}$ and $\mathbf{w}_{h 2}$, respectively $(k=1,2, \ldots, 6)$, then $\operatorname{det}\left(\mathbf{W}^{T} \mathbf{A W}\right)$ can be expressed in the form

$$
\operatorname{det}\left(\mathbf{W}^{T} \mathbf{A W}\right)=\sum_{k=1}^{6} \sum_{p=1}^{6}\left(a_{k} b_{p}-a_{p} b_{k}\right)^{2}
$$

Since $a_{k}$ and $b_{k}$ are linear functions of $\delta_{i 1}$ and $\delta_{i 2}$, the minimum values of $\left(a_{k} b_{p}-a_{p} b_{k}\right)$ in the range $\left[-\Delta_{i 1}, \Delta_{i 1}\right]$ and $\left[-\Delta_{i 2}, \Delta_{i 2}\right]$ can be determined. For $k, p=1, \ldots, 6$, let

$$
\alpha_{k p}=\min \left\{a_{k} b_{p}-a_{p} b_{k} \mid \delta_{i j} \in\left[-\Delta_{i j}, \Delta_{i j}\right], i, j=1,2\right\} .
$$

Then,

$$
\operatorname{det}\left(\mathbf{W}^{T} \mathbf{A W}\right) \geq \sum_{k, p} \alpha_{k p}^{2}=\alpha
$$

Now consider the numerators of $\phi_{1}$ and $\phi_{2}$ in $(10)$ and (11). Since each $\mathbf{w}_{i}$ linearly depends on $\delta_{i j}$, the numerator of $\phi_{1}$ in $(10)$ can be expressed in the form

$$
N\left(\phi_{1}\right)=f\left(\delta_{21}, \delta_{22}\right) g\left(\delta_{11}, \delta_{12}\right)
$$

where $f$ is a quadratic function in $\left(\delta_{21}, \delta_{22}\right)$ and $g$ is a linear function in $\left(\delta_{11}, \delta_{12}\right)$. The maximum values of $f$ and $g$ can be analytically determined. Thus, the maximum values of $N\left(\phi_{1}\right)$ in the range considered can be obtained. The same reasoning applies to the numerator of $\phi_{2}$.

If we denote

$$
\begin{aligned}
& \beta_{1}=\max \left\{\left(\mathbf{w}_{2}^{T} \mathbf{A} \mathbf{w}_{2}\right)\left(\mathbf{w}_{1}^{T} \mathbf{v}_{0}\right)-\left(\mathbf{w}_{1}^{T} \mathbf{A} \mathbf{w}_{2}\right)\left(\mathbf{w}_{2}^{T} \mathbf{v}_{0}\right)\right\} \\
& \beta_{2}=\max \left\{\left(\mathbf{w}_{1}^{T} \mathbf{A} \mathbf{w}_{1}\right)\left(\mathbf{w}_{2}^{T} \mathbf{v}_{0}\right)-\left(\mathbf{w}_{1}^{T} \mathbf{A} \mathbf{w}_{2}\right)\left(\mathbf{w}_{1}^{T} \mathbf{v}_{0}\right)\right\}
\end{aligned}
$$

and

$$
\phi_{1 M}=\frac{\beta_{1}}{\alpha}, \phi_{2 M}=\frac{\beta_{2}}{\alpha}
$$

then, the force magnitude at the two contact points

$$
\phi_{1} \leq \phi_{1 M}, \phi_{2} \leq \phi_{2 M}
$$




\section{Error-Reduction Condition}

Since only small orientational variation is considered, the angular magnitude $\theta$ is small $\left(\leq 5^{\circ}\right)$. Thus, the rotation matrix $\mathbf{R}$ in $\underline{6})$ can be accurately approximated by

$$
\mathbf{R}(\mathbf{u}, \theta)=\mathbf{I}+\sin \theta[\mathbf{u} \times] .
$$

In the following, for an arbitrary wrench (6-D line vector) $\mathbf{w}$, we denote $\mathbf{w}^{\mathbf{u} \times}$ as the cross product operation of $\mathbf{u}$ on $\mathbf{w}$, i.e., if $\mathbf{w}$ has the form

$$
\mathbf{w}=\left[\begin{array}{c}
\mathbf{a} \\
\mathbf{r} \times \mathbf{a}
\end{array}\right]
$$

then,

$$
\mathbf{w}^{\mathbf{u} \times}=\left[\begin{array}{c}
\mathbf{u} \times \mathbf{a} \\
\mathbf{r} \times(\mathbf{u} \times \mathbf{a})
\end{array}\right]
$$

If we denote

$$
\mathbf{d}_{0}^{\prime}=\mathbf{d}_{1}^{\prime}+\mathbf{d}_{b}^{\prime}
$$

and

$$
\mathbf{d}_{0}=\left[\begin{array}{c}
\mathbf{d}_{0}^{\prime} \\
\mathbf{r}_{B} \times \mathbf{d}_{0}^{\prime}
\end{array}\right], \mathbf{d}_{1}=\left[\begin{array}{c}
\mathbf{d}_{1}^{\prime} \\
\mathbf{r}_{B} \times \mathbf{d}_{1}^{\prime}
\end{array}\right], \delta_{1}=\left[\begin{array}{c}
\delta_{1}^{\prime} \\
\mathbf{r}_{B} \times \delta_{1}^{\prime}
\end{array}\right]
$$

then, using $(9)$ and $(14)$, the error-reduction function $(5)$ can be approximated by

$$
\begin{aligned}
F_{\mathrm{er}}(\delta, \theta)= & \left(\mathbf{d}_{0}-\delta_{1}\right)^{T} \mathbf{v}_{0}+\left(\mathbf{d}_{0}-\delta_{1}\right)^{T} \mathbf{A W} \phi \\
& +\left(\mathbf{d}_{1}^{\mathbf{u} \times}\right)^{T}\left(\mathbf{v}_{0}+\mathbf{A W} \phi\right) \sin \theta .
\end{aligned}
$$

For any matrix or vector $\mathbf{Q}$, let $\|\mathbf{Q}\|$ be the conventional norm of $\mathbf{Q}$. Now consider the norm of $\mathbf{d}_{1}^{\mathbf{u} \times}$. Since $\mathbf{u}$ is a unit vector,

$$
\left\|\mathbf{d}_{1}^{\mathbf{u} \times}\right\|=\left\|\left[\begin{array}{c}
\mathbf{u} \times \mathbf{d}_{1}^{\prime} \\
\mathbf{r}_{B} \times\left(\mathbf{u} \times \mathbf{d}_{1}^{\prime}\right)
\end{array}\right]\right\| \leq\left\|\left[\begin{array}{c}
\mathbf{d}_{1}^{\prime} \\
\mathbf{r}_{B} \times \mathbf{d}_{1}^{\prime}
\end{array}\right]\right\|=\left\|\mathbf{d}_{1}\right\| .
$$

If we denote

$$
\begin{array}{cc}
\phi_{M} & =\left[\phi_{1 M}, \phi_{2 M}\right]^{T} \in \mathbb{R}^{2} \\
M & =\left\|\mathbf{d}_{1}^{T}\right\|\left\|\mathbf{v}_{0}\right\|+\left\|\mathbf{d}_{1}^{T} \mathbf{A} \mathbf{W} \phi_{M}\right\|
\end{array}
$$

then, the last term in $\underline{(16)}$

$$
\begin{aligned}
& \left(\mathbf{d}_{1}^{\mathbf{u} \times}\right)^{T}\left(\mathbf{v}_{0}+\mathbf{A W} \boldsymbol{\mathbf { W }}\right) \sin \theta \\
& \leq\left(\left\|\mathbf{d}_{1}^{T}\right\|\left\|\mathbf{v}_{0}\right\|+\left\|\mathbf{d}_{1}^{T} \mathbf{A} \mathbf{W} \phi_{M}\right\|\right) \sin \theta . \\
& \leq M \sin \theta_{M} .
\end{aligned}
$$


Now consider the second term in $\underline{(16)}$

$$
\left(\mathbf{d}_{0}-\delta_{1}\right)^{T} \mathbf{A W} \phi=\left(\mathbf{d}_{0}-\delta_{1}\right)^{T}\left(\mathbf{A w}_{1} \phi_{1}+\mathbf{A} \mathbf{w}_{2} \phi_{2}\right) .
$$

It can be seen that the two functions

$$
g_{i}=\left(\mathbf{d}_{0}-\delta_{1}\right)^{T} \mathbf{A} \mathbf{w}_{i}, i=1,2
$$

are quadratic in $\delta_{i j}$. Thus, the maximum values of $g_{1}$ and $g_{2}$ can be determined analytically. If we denote

$$
M_{i}=\max \left\{g_{i} \mid \delta_{i j} \in\left[-\Delta_{i j}, \Delta_{i j}\right]\right\}, i=1,2
$$

and define a new function

$$
f=\left(\mathbf{d}_{0}-\delta_{1}\right)^{T} \mathbf{v}_{0}+M_{1} \phi_{1 M}+M_{2} \phi_{2 M}+M \sin \theta_{M}(17)
$$

then, for all configurations considered,

$$
F_{\mathrm{er}} \leq f
$$

Since $f$ only contains linear terms in $\left(\delta_{11}, \delta_{12}\right)$, if at the boundary configurations, $\left( \pm \Delta_{11}, \pm \Delta_{12}\right)$, $f<0$, then, for all intermediate configurations, the error-reduction condition is satisfied $F_{\text {er }}<$ 0 . Similarly, if the error-measure vector $\mathbf{d}$ is expressed in terms of $\delta_{2 j}$, the function $f$ will be linear function of $\left(\delta_{21}, \delta_{22}\right)$. The condition $f<0$ at the corresponding boundary configurations $\left( \pm \Delta_{21}, \pm \Delta_{22}\right)$ ensure the error-reduction motion of the body. Thus, we have the following.

Proposition 1: For an $\{f-v, f-v\}$ contact state, if the error-measure vector $\mathbf{d}$ is expressed in terms of $\left(\delta_{i 1}, \delta_{i 2}\right)$, and at the boundary configurations $\left( \pm \Delta_{i 1}, \pm \Delta_{i 2}\right)$, the function $f$ defined in $117)$ is negative, then the admittance will satisfy the error-reduction conditions for all configurations bounded by $\delta_{i j} \in\left[-\Delta_{i j}, \Delta_{i j}\right]$ and $\theta \in\left[0, \theta_{M}\right]$ where $\mathbf{u}$ is arbitrary.

As such, for an $\{f-v, f-v\}$ contact, to ensure error-reducing motion for all configurations within the contact state, four conditions need to be satisfied.

\section{SECTION IV. Sufficient Conditions for $\{v-f, v-f\}$ Contact}

For $\{v-f, v-f\}$ contact, the configuration of the body can be determined by the orientation of the body $(\mathbf{u}, \theta)$ and the location of one of the two contact points $\left(\delta_{i 1}, \delta_{i 2}\right)$.

Suppose that $\theta$ varies within the range of $\left[0, \theta_{M}\right]$, and $\delta_{i j}$ varies within the range of $\left[-\Delta_{i j}, \Delta_{i j}\right]$. We prove that, if an admittance matrix $\mathbf{A}$ satisfies a set of conditions determined at the "boundary" configurations, then the same admittance will ensure that the motion is errorreducing for any intermediate configuration $\theta \in\left[0, \theta_{M}\right], \delta_{i j} \in\left[-\Delta_{i j}, \Delta_{i j}\right]$. 
To prove the results, we first consider configuration variation in orientation and translation separately. Then, by combining the two cases, general results are obtained.

\section{A. Configuration Variation in Orientation}

Consider only orientational variation of the contact configuration as illustrated in Fig. 3(a). Since the positions of the two contact points are constant in the body frame, we assume that the location of one contact vertex of the held body is constant in the face plane, and both the direction of the error-reduction vector $\mathbf{d}$ and the direction of the contact force are changed by changing the orientation. We prove that, for $\theta_{M} \leq \frac{\pi}{10}$, if $\mathbf{A}$ satisfies a set of conditions at $\theta=0$ (defined at a central orientation), then an error-reducing motion is ensured for all configurations obtained by rotating about an arbitrary axis $\mathbf{u}$ with angle $\theta \leq \theta_{M}$.

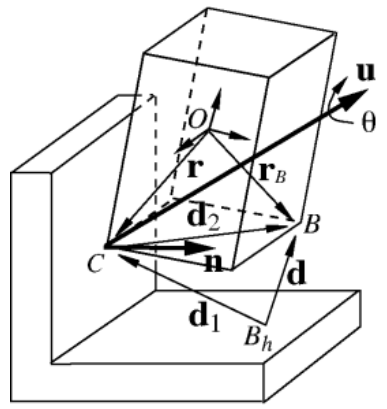

(a)

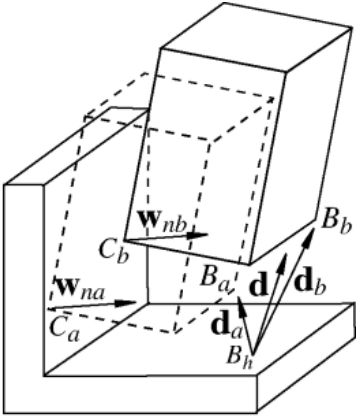

(b)

Fig. 3. Vertex-face contact and the coordinate frame.

Let $\mathbf{w}_{0}$ be the contact wrench and $\mathbf{d}_{0}$ be the position vector associated with $\theta=0$. Suppose that at $\theta=0$, an error-reducing motion is obtained, i.e.,

$$
\mathbf{d}_{0}{ }^{T} \mathbf{v}_{0}+\mathbf{d}_{0}{ }^{T} \mathbf{A} \mathbf{w}_{0}<0
$$

Consider a rotation given by an angle change $\theta \in\left[0, \theta_{M}\right]$ about an axis $\mathbf{u}$. If we denote $\mathbf{n}_{0}$ as the surface normal associated with $\theta=0$, then in the body coordination frame, the surface normal associated with varying $(\mathbf{u}, \theta)$ at contact $i$ is

$$
\mathbf{n}_{i}=\mathbf{R}(\theta) \mathbf{n}_{i 0}
$$

where $\mathbf{R}$ is the rotation matrix having the form of (6).

Since contact is frictionless, the contact force is along the surface normal at the contact point. Thus, the unit contact wrench is

$$
\mathbf{w}_{i}(\theta)=\left[\begin{array}{c}
\mathbf{n}_{i} \\
\mathbf{r}_{i} \times \mathbf{n}_{i}
\end{array}\right]=\left[\begin{array}{c}
\mathbf{R n}_{i 0} \\
\mathbf{r}_{i} \times \mathbf{R} \mathbf{n}_{i 0}
\end{array}\right]
$$


where $\mathbf{r}_{i}$ is the position vector from the origin of the body frame to the contact point $i$ (constant in body frame).

Since the orientational variation considered corresponds to pure rotation about one contact point $C$, the error-measure three-vector $\mathbf{d}^{\prime}$ for an intermediate configuration can be expressed in the body frame as

$$
\mathbf{d}^{\prime}=\mathbf{R d}_{1}^{\prime}+\mathbf{d}_{2}^{\prime}
$$

where $\mathbf{d}_{1}^{\prime}$ is the position three-vector from $B_{h}$ to the contact point $C$ and $\mathbf{d}_{2}^{\prime}$ is the position three-vector from $C$ to point $B$. Note that $\mathbf{d}_{1}^{\prime}$ is a constant in the global frame and $\mathbf{d}_{2}^{\prime}$ is constant in the body frame. Then, in the body frame, the line vector associated with $\mathbf{d}^{\prime}$ is obtained as

$$
\mathbf{d}(\theta)=\left[\begin{array}{c}
\mathbf{d}^{\prime} \\
\mathbf{r}_{B} \times \mathbf{d}^{\prime}
\end{array}\right]=\left[\begin{array}{c}
\mathbf{R} \mathbf{d}_{1}^{\prime} \\
\mathbf{r}_{B} \times \mathbf{R} \mathbf{d}_{1}^{\prime}
\end{array}\right]+\left[\begin{array}{c}
\mathbf{d}_{2}^{\prime} \\
\mathbf{r}_{B} \times \mathbf{d}_{2}^{\prime}
\end{array}\right]
$$

where $\mathbf{r}_{B}$ is the position vector from the body frame origin to point $B$.

Using the rotation matrix in $\underline{(14)}$, the contact wrench $\underline{(20)}$ and the error-measure vector $\underline{(21)}$ can be accurately approximated by

$$
\begin{gathered}
\mathbf{w}_{i}=\mathbf{w}_{0 i}+\mathbf{w}_{0 i}^{\mathbf{u} \times} \sin \theta \\
\mathbf{d}=\mathbf{d}_{0}+\mathbf{d}_{1}^{\mathbf{u} \times} \sin \theta
\end{gathered}
$$

where $\mathbf{d}_{0}$ is the line vector associated with vector $\mathbf{d}_{1}^{\prime}+\mathbf{d}_{2}^{\prime}$ and position $\mathbf{r}_{B}$.

Substituting $\underline{(22)}$ and $\underline{(23)}$ into $F_{\text {er }} \underline{(5)}$ yields

$$
F_{\mathrm{er}}=F(0)_{\mathrm{er}}+F_{1} \sin \theta+F_{2} \sin ^{2} \theta+\cdots+F_{5} \sin ^{5} \theta(24)
$$

where $F_{i}$ is a function involving $\mathbf{w}_{0 i}^{\mathbf{u} \times}$ and $\mathbf{d}_{1}^{\mathbf{u} \times}$. Since $\mathbf{u}$ is a unit vector, $\left\|\mathbf{w}_{0 i}^{\mathbf{u} \times}\right\| \leq\left\|\mathbf{w}_{0 i}\right\|$ and $\|$ $\mathbf{d}_{1}^{\mathbf{u} \times}\|\leq\| \mathbf{d}_{1} \|$. Thus, the bound for $F_{i}$ can be determined.

For small $\theta \leq 5^{\circ}$, terms containing $\sin ^{m} \theta$ for $m \geq 2$ can be neglected. For this approximation, only the bound on $F 1$ is needed. By explicit calculation, $F_{1}$ can be expressed in the form

$$
F_{1}=\mathbf{d}_{1}^{\mathbf{u} \times} \cdot \mathbf{v}_{0}+\sum_{i, j} c_{i j}\left(\mathbf{w}_{i}^{T} \mathbf{A} \mathbf{w}_{j}^{\mathbf{u} \times}\right)
$$

where each $c_{i j}$ is a function of the admittance $\mathbf{A}$. Thus, 


$$
\begin{aligned}
\left|F_{1}\right| & =\left|\mathbf{d}_{1}^{\mathbf{u} \times} \cdot \mathbf{v}_{0}\right|+\sum_{i, j}\left|c_{i j}\right| \cdot\left|\mathbf{w}_{i}^{T} \mathbf{A} \mathbf{w}_{j}^{\mathbf{u} \times}\right| \\
& \leq\left\|\mathbf{d}_{1}\right\| \cdot\left\|\mathbf{v}_{0}\right\|+\sum_{i, j}\left|c_{i j}\right| \cdot\left\|\mathbf{w}_{i}\right\| \cdot\|\mathbf{A}\| \cdot\left\|\mathbf{w}_{j}\right\| \cdot \\
& =F_{1 M} .
\end{aligned}
$$

Therefore, if the condition

$$
F_{\mathrm{er}}(0)+F_{1 M} \sin \theta_{M}<0(25)
$$

is satisfied, then the error-reduction is achieved for any $\theta \in\left[0, \theta_{M}\right]$ and $\mathbf{u}$ in any direction.

\section{B. Configuration Variation in Translation}

In this case, only translation in the contact face is allowed. Thus, the contact force does not change in the body frame, and the error-measure vector $d$ at an intermediate configuration is a convex combination of $\mathbf{d}$ 's measured at the four boundary points. Similar to the single PC $\{v-f\}$ case [6], it can be proved that if at two configurations $\left(-\Delta_{1}, \delta_{2}\right)$ and $\left(\Delta_{1}, \delta_{2}\right)$, the errorreduction condition is satisfied, then the error-reduction condition must be satisfied for all intermediate configurations $\left(\delta_{1}, \delta_{2}\right)$ with $\delta_{1} \in\left[-\Delta_{1}, \Delta_{1}\right]$. The same result holds true for variation in $\delta_{2}$ while $\delta_{1}$ is constant.

\section{General Case}

Using the same procedure presented in [4], the results presented in Section IV-A and Section IV$B$ can be generalized to intermediate vertex-face contact configurations involving both translational and orientational variations from configurations at which the conditions were imposed. Thus, we have the following.

Proposition 2: For a $\{v-f, v-f\}$ contact state with variation of orientation $\left[0, \theta_{M}\right]$ and variation of translation $\left[-\Delta_{i j}, \Delta_{i j}\right]$, if inequality $\underline{(25)}$ is satisfied at the translational boundary points $\left( \pm \Delta_{11}, \pm \Delta_{12}\right),\left( \pm \Delta_{21}, \pm \Delta_{22}\right)$, then the admittance will satisfy the error-reduction condition for all configurations bounded by $\delta_{i j} \in\left[-\Delta_{i j}, \Delta_{i j}\right]$, and $\theta \in\left[0, \theta_{M}\right]$ in any rotation direction.

Therefore, for a $\{v-f, v-f\}$ contact state, to ensure that the motion response due to contact is error reducing for all configurations considered, eight conditions need be satisfied.

\section{SECTION V. Sufficient Conditions for $\{f-v, v-f\}$ Contact}

Later, for $\{f-v, v-f\}$ contact, a set of conditions are identified that, when satisfied for a given admittance matrix $\mathbf{A}$ at the "boundary" points, ensures error-reducing motion for all intermediate configurations $\theta \in\left[0, \theta_{M}\right], \delta_{i} \in\left[-\Delta_{i}, \Delta_{i}\right]$. The procedure is similar to that for the $\{f-v, f-v\}$ case. 
First, the bounds for the contact force magnitudes are estimated. Then, based on the bounds contact force magnitudes, a new linear function $F$ is constructed such that it bounds the errorreduction functions $F_{\text {er }} \leq F$ for all configurations. By evaluating the function $F$ at the boundary configurations, the set of conditions on the admittance for error reduction is obtained.

To determine the bounds of contact force magnitudes, $\underline{(12)}$ is used. The denominator of $\phi_{i}$ in (10) and (11), $D\left(\phi_{i}\right)$, can be expressed as

$$
D\left(\phi_{i}\right)=\operatorname{det}\left(\mathbf{W}^{T} \mathbf{A W}\right)=\sum\left(a_{k} b_{p}-a_{p} b_{k}\right)^{2} .
$$

For $\{f-v\}$ contact, the contact wrench has the form

$$
\mathbf{w}_{1}=\left[\begin{array}{c}
\mathbf{n}_{1} \\
\left.\left(\mathbf{r}_{01}+\delta_{11} \mathbf{b}_{11}+\delta_{12} \mathbf{b}_{12}\right) \times \mathbf{n}_{1}\right] \cdot(26)
\end{array}\right.
$$

For $\{v-f\}$ contact, the contact wrench has the form

$$
\mathbf{w}_{2}=\left[\begin{array}{c}
\mathbf{R} \mathbf{n}_{2} \\
\mathbf{r}_{2} \times \mathbf{R n}_{2}
\end{array}\right] .
$$

Let $\mathbf{w}_{20}$ be the unit contact wrench (constant) at the initial configuration. Using (14), $\mathbf{w}_{2}$ can be written as

$$
\mathbf{w}_{2}=\mathbf{w}_{20}+\mathbf{w}_{20}^{\mathbf{u} \times} \sin \theta \cdot(27)
$$

Substituting $(26)$ and $(27)$ into $(12)$, the corresponding $a_{k}$ and $b_{k}$ have the form

$$
\begin{aligned}
& a_{k}=a_{k 1} \delta_{11}+a_{k 2} \delta_{12}+a_{i 0} \\
& b_{k}=b_{k 1}(\mathbf{u}) \sin \theta+b_{k 0} .
\end{aligned}
$$

Thus, $f_{k p}=\left(a_{k} b_{p}-a_{p} b_{k}\right)$ can be written in the form

$$
f_{k p}=\left(c_{1} \delta_{11}+c_{2} \delta_{12}+c_{3}\right) \sin \theta+c_{0}(30)
$$

where the coefficients ci are functions of the admittance $\mathbf{A}$ and rotation variable $\mathbf{u}$.

For any given rotation direction $\mathbf{u}$, the maximum and minimum values of $f_{k p}$ occur at the boundary points of the configurations considered. Let $P_{B}$ be the set of all boundary points. Then, at a point $\left(\delta_{i m}, \theta_{m}\right) \in P_{B}, f_{k p}$ is a linear function of $\mathbf{u}$. Thus, the maximum and minimum of $f_{k p}\left(\delta_{i m}, \theta_{m}\right)$ can be determined.

Let $f_{m_{k p}}$ be the minimum of $\left|f_{k p}\left(\delta_{i m}, \theta_{m}\right)\right|$ for all $\mathbf{u}$ at $\left(\delta_{i m}, \theta_{m}\right)$ and let

$$
\alpha_{k p}=\min \left\{f_{m_{k p}},(\delta, \theta) \in P_{B}\right\}
$$

then, 


$$
\operatorname{det}\left(\mathbf{W}^{T} \mathbf{A W}\right) \geq \sum \alpha_{k p}^{2}=\alpha
$$

Consider $\phi_{1}$ in (10). Using $\underline{(26)}$ and $\underline{(27)}$, the numerator of $\phi_{1}, N\left(\phi_{1}\right)$, can be expressed as

$$
\begin{array}{cc}
N\left(\phi_{1}\right) \quad=\left(\lambda_{1} \sin ^{2} \theta+\lambda_{2} \sin \theta+\lambda_{3}\right) \delta_{11} \\
+\left(\lambda_{4} \sin ^{2} \theta+\lambda_{5} \sin \theta+\lambda_{6}\right) \delta_{12}+\lambda_{0}
\end{array}
$$

where the coefficients $\lambda_{i}$ are functions of the admittance $\mathbf{A}$. The maximum value of $N\left(\phi_{1}\right), \beta_{1}$, can be determined.

Similarly, the numerator of $\phi_{2}, N\left(\phi_{2}\right)$, can be expressed as

$$
\begin{gathered}
N\left(\phi_{2}\right)=\left(\xi_{1} \delta_{11}^{2}+\xi_{2} \delta_{12}^{2}+\xi_{3} \delta_{11} \delta_{12}+\xi_{4} \delta_{11}+\xi_{5} \delta_{12}\right) \sin \theta \\
+\xi_{0}
\end{gathered}
$$

where each coefficient $\xi_{i}$ is a function of $\mathbf{A}$ and linearly depends on $\mathbf{u}$. The maximum value of $N\left(\phi_{2}\right), \beta_{2}$, can be determined.

Let

$$
\phi_{1 M}=\frac{\beta_{1}}{\alpha}, \phi_{2 M}=\frac{\beta_{2}}{\alpha}
$$

then, for any configuration,

$$
\phi_{1} \leq \phi_{1 M}, \phi_{2} \leq \phi_{2 M}
$$

Now consider the term: $f_{i}=\mathbf{d}^{T} \mathbf{A} \mathbf{w}_{i}$. Based on the $\{f-v\}$ contact point $C$, the error-measure vector $\mathbf{d}$ can be expressed as a function of $\left(\delta_{11}, \delta_{12}\right)$ and $(\mathbf{u}, \theta)$

$$
\mathbf{d}=\mathbf{d}_{0}+\mathbf{d}_{1}^{\mathbf{u} \times} \sin \theta-\delta_{1}
$$

where $\mathbf{d}_{0}$ is the line vector defined in $\underline{(23)}$. Substituting the aforementioned equation and $\underline{(27)}$ into $f_{2}$ yields

$$
\begin{aligned}
f_{2}= & \left(\mathbf{d}_{0}+\mathbf{d} \mathbf{u} \times \sin \theta-\delta_{1}\right)^{T} \mathbf{A}\left(\mathbf{w}_{20}+\mathbf{w}_{20}^{\mathbf{u} \times} \sin \theta\right) \\
= & \left(\mathbf{d}_{1}^{\mathbf{u} \times} \mathbf{A} \mathbf{w}_{20}+\mathbf{d}_{0}^{T} \mathbf{A} \mathbf{w}_{20}^{\mathbf{u} \times}-\delta_{1}^{T} \mathbf{A} \mathbf{w}_{20}^{\mathbf{u} \times}\right) \sin \theta \\
& +\mathbf{d}_{1}^{\mathbf{u} \times} \mathbf{A} \mathbf{w}_{20}^{\mathbf{u} \times} \sin ^{2} \theta+\left(\mathbf{d}_{0}-\delta_{1}\right)^{T} \mathbf{A} \mathbf{w}_{20} .
\end{aligned}
$$

If we denote

$$
\begin{gathered}
M_{1}=\left\|\mathbf{d}_{1}\right\| \cdot\left\|\mathbf{A} \mathbf{w}_{20}\right\| \\
M_{2}=\left(\left\|\mathbf{d}_{1}\right\|+\left\|\mathbf{d}_{0}\right\|+\delta_{1 M}\right)\left\|\mathbf{A} \mathbf{w}_{20}\right\|
\end{gathered}
$$

where $\delta_{1 M}$ is the maximum value of $\left\|\delta_{1}\right\|$, then,

$$
f_{2} \leq\left(\mathbf{d}_{0}-\delta_{1}\right)^{T} \mathbf{A} \mathbf{w}_{20}+M_{1} \sin ^{2} \theta_{M}+M_{2} \sin \theta_{M}
$$


Similarly, for $f_{1}$, we have

$$
\begin{gathered}
f_{1}=\left(\mathbf{d}_{0}+\mathbf{d}_{1}^{\mathbf{u} \times} \sin \theta-\delta_{1}\right)^{T} \mathbf{A} \mathbf{w}_{1} \\
=\left(\mathbf{d}_{0}-\delta_{1}\right)^{T} \mathbf{A} \mathbf{w}_{1}+\left(\mathbf{d}_{1}^{\mathbf{u} \times}\right)^{T} \mathbf{A} \mathbf{w}_{1} \sin \theta .
\end{gathered}
$$

Since $\mathbf{w}_{1}$ linearly depends on $\left(\delta_{11}, \delta_{12}\right)$, the maximum value of $\left\|\mathbf{w}_{1}\right\|$ can be determined on the boundary of $\left(\delta_{11}, \delta_{12}\right)$. Let $w_{1 M}=\max \left\{\left\|\mathbf{w}_{1}\right\|\right\}$ and $M_{3}=\left\|\mathbf{d}_{1}\right\| \cdot\|\mathbf{A}\| w_{1 M}$, then,

$$
f_{1} \leq\left(\mathbf{d}_{0}-\delta_{1}\right)^{T} \mathbf{A} \mathbf{w}_{1}+M_{3} \sin \theta_{M}
$$

Now consider the function defined by

$$
\begin{aligned}
F \quad & =\left[\left(\mathbf{d}_{0}-\delta_{1}\right)^{T} \mathbf{A} \mathbf{w}_{20}+M_{1} \sin ^{2} \theta_{M}+M_{2} \sin \theta_{M}\right] \phi_{1 M} \\
& +\left[\left(\mathbf{d}_{0}-\delta_{1}\right)^{T} \mathbf{A} \mathbf{w}_{1}+M_{3} \sin \theta_{M}\right] \phi_{2 M}+\left(\mathbf{d}_{0}-\delta_{1}\right)^{T} \mathbf{v}_{0}
\end{aligned}
$$

Then, for a configuration in the considered range,

$$
F_{\text {er }} \leq F .(32)
$$

Thus, if the condition $F \leq 0$ is satisfied, then the error-reduction motion is ensured for all configurations considered. Note $F$ in (31) is a quadratic polynomial in $\left(\delta_{11}, \delta_{12}\right)$, and sufficient conditions for $F<0$ can be obtained.

Consider the linear equations defined by

$$
\frac{\partial F}{\partial \delta_{11}}=0, \frac{\partial F}{\partial \delta_{12}}=0 .
$$

Let $\delta_{11}^{\prime}$ and $\delta_{12}^{\prime}$ be the root of the aforementioned equations. Then, the following conditions ensure that the function $F$ does not change sign in the range of $\left(-\Delta_{11}, \Delta_{11}\right),\left(-\Delta_{12}, \Delta_{12}\right)$

$$
\left|\delta_{11}^{\prime}\right|>\Delta_{11},\left|\delta_{12}^{\prime}\right|>\Delta_{12} \cdot(33)
$$

Therefore, we have the following.

Proposition 3: For an $\{f-v, v-f\}$ contact state, if: 1 ) at any one of the boundary points $\left( \pm \Delta_{11}, \pm \Delta_{12}\right)$, the function $F$ defined in (31) is negative and 2 ) conditions in (33) are satisfied, then the admittance will satisfy the error-reduction condition for all configurations bounded by $\delta_{i j} \in\left[-\Delta_{i j}, \Delta_{i j}\right]$ and rotation in an arbitrary direction with angle $\theta \leq \theta_{M}$.

As such, for a $\{f-v, v-f\}$ contact state, to ensure that the motion response due to contact is error reducing for all configurations considered, four conditions need to be satisfied. 


\section{SECTION VI. Sufficient Conditions for $\{e-e, e-e\}$ Contact}

In order to obtain the error-reduction function, the contact wrench and the error-measure vector $\mathbf{d}$ are first expressed in terms of $\delta_{i}$ and $\theta$. Similar to the process described in Section $\mathrm{V}$, a new function $F$ is constructed to bound the error-reduction function $F_{\mathrm{er}}$. Since the constructed function linearly depends on the the configuration coordinates $\delta_{i j}$, error-reduction conditions are obtained by evaluating the function at the boundary configurations.

For an edge-edge cross contact state as shown in Fig. 4, the direction of the contact force is along the common normal of the two edges. Let $\mathbf{e}_{1}$ and $\mathbf{e}_{2}$ be the two unit vectors along the two edges, respectively, then, the direction of the force must be $\mathbf{n}=\mathbf{e}_{1} \times \mathbf{e}_{2}$. Note that $\mathbf{e}_{1}$ is constant in the body frame while $\mathbf{e}_{2}$ is constant in the global frame. When the held body rotates relative to the fixtured body about the contact point $C$, the vector $\mathbf{e}_{2}$ in the body frame can be expressed as $\mathbf{R e}_{2}$ where $\mathbf{R}$ is the rotation matrix. When the held body translates relative to the fixtured body along $\mathbf{e}_{1}$ as shown in Fig. 4 , the description of the contact wrench changes in a body-based coordinate frame as the contact point changes (although its direction is constant). Thus, the contact wrench is a function involving both the translational and orientational variables $\left(\delta_{i 1}, \delta_{i 2}, \theta\right)$.

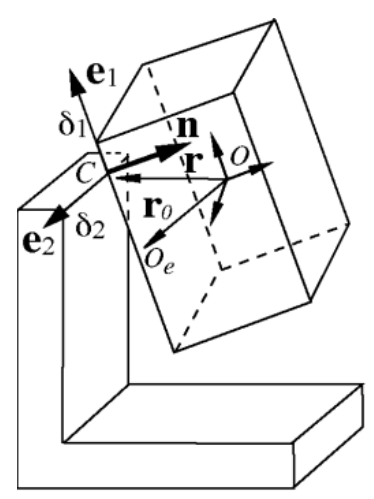

(a)

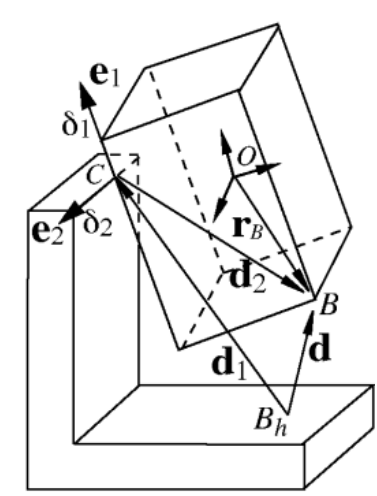

(b)

Fig. 4. Edge-edge cross contact and the coordinate frame.

For all edge-edge cross contact cases, the direction of the force depends only on the orientational variation while the position vector of the contact point $\mathbf{r}_{i}$ depends only the translational variation along the contact edge of the held body $\mathbf{e}_{i 1}$. For arbitrary $\left(\delta_{i 1}, \delta_{i 2}\right), \mathbf{r}_{i}$ can be expressed as

$$
\mathbf{r}_{i}=\mathbf{r}_{i 0}+\delta_{i 1} \mathbf{e}_{i 1}
$$

where $\mathbf{r}_{i 0}$ is a vector from the body frame to a centrally located point on the edge $\mathbf{e}_{i 1}$ (constant). The unit wrench corresponding to the surface normal has the form of (8).

It can be seen that the direction of $\mathbf{w}_{1}$ is determined by $\mathbf{e}_{11}$ and $\mathbf{e}_{12}$ and the last component (the moment term) is a linear function of $\delta_{11}$. 
Let $\mathbf{d}_{1}^{\prime}$ and $\mathbf{d}_{2}^{\prime}$ be the two vectors from $B_{h}$ to $C$ and from $C$ to $B$ for $(\delta, \theta)=(0,0)$, respectively, then for arbitrary $\left(\delta_{11}, \delta_{12}\right)$ with $\theta=0$, the error-measure vector $\mathbf{d}^{\prime}$ is

$$
\mathbf{d}(\delta)_{1}^{\prime}=\mathbf{d}_{1}^{\prime}+\mathbf{d}_{2}^{\prime}+\delta_{11} \mathbf{e}_{11}+\delta_{12} \mathbf{e}_{12}, \delta_{1 i} \in\left[-\Delta_{1 i}, \Delta_{1 i}\right]
$$

Note that $\mathbf{d}_{1}^{\prime}$ and $\mathbf{e}_{12}$ are constant in the global coordinate frame while $\mathbf{d}_{2}^{\prime}$ and $\mathbf{e}_{11}$ are constant in the body frame. Thus, for an arbitrary orientation $(\mathbf{u}, \theta)$ and $\delta_{1 i} \in\left[-\Delta_{1 i}, \Delta_{1 i}\right]$, the error-measure three-vector $\mathbf{d}^{\prime}$ is a function of $(\mathbf{u}, \theta)$ and $\delta_{1 i}$ having the form

$$
\mathbf{d}^{\prime}(\mathbf{u}, \theta, \delta)=\mathbf{R}\left(\mathbf{d}_{1}^{\prime}+\delta_{12} \mathbf{e}_{12}\right)+\mathbf{d}_{2}^{\prime}+\delta_{11} \mathbf{e}_{11}
$$

where $\mathbf{R}$ is the rotation matrix.

If we denote

$$
\delta_{i 1}=\delta_{i 1}\left[\begin{array}{c}
\mathbf{e}_{i 1} \\
\mathbf{r}_{i B} \times \mathbf{e}_{i 1}
\end{array}\right], \delta_{i 2}=\delta_{i 2}\left[\begin{array}{c}
\mathbf{e}_{i 2} \\
\mathbf{r}_{i B} \times \mathbf{e}_{i 2}
\end{array}\right]
$$

and denote $\mathbf{d}_{i}$ as the line vectors associated with $\mathbf{d}_{i}^{\prime}$, then the error-measure function $\mathbf{d}$ can be expressed as

$$
\mathbf{d}=\mathbf{R}\left(\mathbf{d}_{1}+\delta_{12}\right)+\mathbf{d}_{2}+\delta_{11}
$$

For rotation $\mathbf{R}$, the direction of the force at $i$ is

$$
\mathbf{n}_{i}=\mathbf{e}_{i 1} \times \mathbf{R e}_{i 2}, i=1,2 .
$$

The unit contact wrench can be expressed as

$$
\mathbf{w}_{i}=\left[\begin{array}{c}
\mathbf{e}_{i 1} \times \mathbf{R} \mathbf{e}_{i 2} \\
\mathbf{r}_{i} \times\left(\mathbf{e}_{i 1} \times \mathbf{R} \mathbf{e}_{i 2}\right)
\end{array}\right]
$$

Using (14), the aforementioned equation can be written as

$$
\mathbf{w}_{i}=\left[\begin{array}{c}
\mathbf{e}_{i 1} \times \mathbf{e}_{i 2} \\
\mathbf{r}_{i} \times\left(\mathbf{e}_{i 1} \times \mathbf{e}_{i 2}\right)
\end{array}\right]+\sin \theta\left[\begin{array}{c}
\mathbf{e}_{i 1} \times\left(\mathbf{u} \times \mathbf{e}_{i 2}\right) \\
\mathbf{r}_{i} \times\left[\mathbf{e}_{i 1} \times\left(\mathbf{u} \times \mathbf{e}_{i 2}\right)\right]
\end{array}\right]
$$

Substituting the aforementioned expressions into the error-reduction function (5), we have

$F_{\mathrm{er}}=F_{0}+F_{1} \sin \theta+F_{2} \sin ^{2} \theta+\cdots+F_{5} \sin ^{5} \theta$

where $F_{i}$ are functions of $\mathbf{A}$ and $\mathbf{u}$.

For small $\leq 5^{\circ}$, higher orders than $\sin ^{2} \theta$ can be neglected. Thus, the error-reduction function can be expressed in the form

$$
F_{\mathrm{er}}=F_{0}+F_{1} \sin \theta+F_{2} \sin ^{2} \theta
$$

where 


$$
\begin{aligned}
& F_{0}=c_{0}+\sum c_{i j} \delta_{i j} \\
& F_{i}=F_{i}(\delta, \mathbf{u}), i=1,2
\end{aligned}
$$

Using the same process in Section IV-A, the bounds for $F_{1}$ and $F_{2}$ can be obtained

$$
F_{1} \leq M_{1}, F_{2} \leq M_{2}
$$

Consider the function defined by

$$
F=F_{0}+M_{1} \sin \theta_{M}+M_{2} \sin ^{2} \theta_{M} .
$$

Then, for any configuration, $F_{\text {er }} \leq F$. Note that $F$ is a linear function in $\delta_{i j}$. Therefore, if at the boundary points $\delta_{i j M}$, then for all intermediate configuration, the error-reduction motion is ensured. Thus, we have the following.

Proposition 4: For a $\{e-e, e-e\}$ contact state, if at the boundary points $\left( \pm \Delta_{i 1}, \pm \Delta_{i 2}\right)$, the function $F$ defined in (35) is negative, then the admittance will satisfy the error-reduction condition for all configurations bounded by $\delta_{i j} \in\left[-\Delta_{i j}, \Delta_{i j}\right]$ and rotation in an arbitrary direction with angle $\theta \leq \theta_{M}$.

Therefore, for a $\{e-e, e-e\}$ contact state, to ensure that the motion response due to contact is error reducing for all configurations considered, eight conditions need to be satisfied.

\section{SECTION VII. Discussion and Summary}

In Sections III, VI, admittance selection conditions for four typical two-PC states each in singlepoint contact were presented. The strategies and procedures can be used for any combination of two single-contact PCs. In general, for each PC, translational variables $\delta_{i j}$ are chosen based on the PC's type. Using translational variables $\delta_{i j}$ and orientational variables $(\mathbf{u}, \theta)$, the contact wrenches $\mathbf{w}_{i}$ and error-measure vector $\mathbf{d}$ are obtained and the interaction of the wrenches from the two PCs is addressed in the calculation of their magnitudes. Then, by (5), the errorreduction function is expressed in terms of $\left(\delta_{i j}, \mathbf{u}, \theta\right)$. The function obtained is a polynomial in $\left(\delta_{i j}, \sin \theta\right)$ with coefficients being functions of $\mathbf{u}$ and the admittance $\mathbf{A}$. Based on the nature of the contact and the properties of the error-reduction function, selection conditions for the admittance are obtained.

Sections III, VI present results for four of the six combinations of two single-point contact PCs. The remaining two combinations are similar. Note that both the wrench associated with $\{e-e\}$ contact $(34)$ and the wrench associated with $\{\{v-f\}$ contact $(22)$ contain only the linear term in $\sin \theta$. Thus, for $\{f-v, e-e\}$ contact, the approach presented in Section $V$ for $\{f-v, v-f\}$ contact can be used. Similarly, the approach presented in Section VI for $\{e-e, e-e\}$ contact can be used for $\{v-f, e-e\}$ contact.

The admittance selection conditions are obtained by geometric and force analysis of each contact state. Redundant coordinates $\delta_{i j}$ are used to describe the translational variation. These 
coordinates are treated as independent variables in a large range (without considering the constraints due to contact). Thus, the conditions obtained are conservative. To make the conditions less conservative, the range of configuration variables considered can be decomposed into a number of nonoverlapping subranges, each addressed with equivalent conditions.

In this paper, a single admittance control law $(1)$ is considered for each contact state. The sufficient conditions obtained impose conditions on the admittance to ensure error reducing motion for the entire contact state. Due to uncertainty in identifying which contact state actually occurs, a single admittance control law (1)could be used for all contact cases, if the conditions for all contact states were satisfied simultaneously.

In practice, the selection of an appropriate admittance $\mathbf{A}$ can be formulated as a search routine to find an admittance matrix $\mathbf{A}$ subject to the appropriate conditions. For instance, an optimization procedure can be used to find an admittance matrix for which sufficient conditions such as those described in the paper are used as "constraints." An example illustrating the application of error-reduction conditions to obtain a desired admittance was presented in [5] for a planar case. This procedure applies to the spatial cases as well.

In summary, we have presented conditions for admittance selection of a polyhedral rigid body for force-guided assembly in cases having two point contact PCs. We have shown that, for these cases, the admittance control law can be selected based on its behavior at a finite number of configurations. If the error-reduction conditions are satisfied at these configurations, the error reduction conditions will be satisfied for all intermediate configurations.

\section{References}

1J. M. Schimmels, M. A. Peshkin, "Admittance matrix design for force guided assembly", IEEE Trans. Robot. Autom., vol. 8, no. 2, pp. 213-227, Apr. 1992.

${ }^{2} \mathrm{~S}$. Huang, J. M. Schimmels, "Achieving an arbitrary spatial stiffness with springs connected in parallel", ASME J. Mech. Des., vol. 120, no. 4, pp. 520-526, Dec. 1998.

${ }^{3} \mathrm{~N}$. Hogan, "Impedance control: An approach to manipulator. Parts I-III", ASME J. Dyn. Syst. Meas. Control, vol. 107, no. 1, pp. 1-24, 1985.

${ }^{4}$ S. Huang, J. M. Schimmels, "Sufficient conditions used in admittance selection for force-guided assembly of polygonal parts", IEEE Trans. Robot. Autom., vol. 19, no. 4, pp. 737-742, 2003.

${ }^{5}$ S. Huang, J. M. Schimmels, "Admittance selection for force-guided assembly of polygonal parts despite friction", IEEE Trans. Robot., vol. 20, no. 5, pp. 817-829, Oct. 2004.

${ }^{6} \mathrm{~S}$. Huang, J. M. Schimmels, "Spatial admittance selection conditions for frictionless force-guided assembly of polyhedral parts in single principal contact", IEEE Trans. Robot., vol. 22, no. 2, pp. 225-239, Apr. 2006.

7J. M. R. Martinez, J. Duffy, "On the metric of rigid body displacements for infinite and finite bodies", ASME J. Mech. Des., vol. 117, no. 1, pp. 41-47, 1995.

${ }^{8}$ D. E. Whitney, "Quasi-static assembly of compliantly supported rigid parts", ASME J. Dyn. Syst. Meas. Control, vol. 104, no. 1, pp. 65-77, 1982. 
${ }^{9}$ M. A. Peshkin, "Programmed compliance for error-corrective manipulation", IEEE Trans. Robot. Autom., vol. 6, no. 4, pp. 473-482, 1990.

${ }^{10} \mathrm{H}$. Asada, "Teaching and learning of compliance using neural net", IEEE Trans. Robot. Autom., vol. 9, no. 6, pp. 863-867, 1993.

${ }^{11} \mathrm{M}$. Shimizu, K. Kosuge, "An admittance design method for general spatial parts mating", Proc. IEEE Int. Conf. Robot. Autom., vol. 4, pp. 3571-3576, 2004.

${ }^{12}$ M. S. Ohwovoriole, B. Roth, "An extension of screw theory", ASME J. Mech. Des., vol. 103, no. 4, pp. 725-735, 1981.

13. Xiao, L. Zhang, "Contact constraint analysis and determination of geometrically valid contact formations from possible contact primitives", IEEE Trans. Robot. Autom., vol. 13, no. 3, pp. 456466, Jun. 1997.

${ }^{14}$ R. M. Murray, Z. Li, S. S. Sastry, A Mathematical Introduction to Robotic Manipulation, FL, Boca Raton:CRC Press, 1994. 